\title{
La utilidad de la ultrasonografía como herramienta diagnóstica para detectar artritis psoriásica en pacientes con psoriasis
}

\author{
Tomás Cazenave ${ }^{1}$, Christian Waimann ${ }^{2}$, Gustavo Citera ${ }^{1}$, Clara Latorre$^{3}$, Javier Ubogui ${ }^{3}$, Miriam Saposnik ${ }^{3}$, Ethel \\ Saturansky ${ }^{3}$, Marcos Rosemffet ${ }^{1}$
}

${ }^{1}$ Instituto de Rehabilitación Psicofísica, Buenos Aires. ${ }^{2}$ Hospital Hector Cura, Olavarría, Buenos Aires. ${ }^{3}$ Psoriahué, Buenos Aires. Instituto de Rehabilitación Psicofísica, Echeverría 955, CABA.

\begin{abstract}
Resumen
El objetivo de nuestro estudio fue evaluar la prevalencia de entesopatía subclínica en pacientes con psoriasis cutánea (Ps) utilizando un score ultrasonográfico (US) como herramienta diagnóstica en la práctica diaria y evaluar la habilidad de dicho score para discriminar pacientes con Ps y Artritis Psoriásica (APs). Material y métodos: Se estudiaron pacientes con diagnóstico de Ps (según diagnóstico por dermatólogo), APs (según criterios CASPAR) y controles sanos (CS). Se evaluaron diez sitios de entesis (de manera bilateral): inserción del tendón del cuádriceps, tendón proximal y distal del ligamento patelar, tendón de Aquiles y fascia plantar. La US identificó Ios siguientes hallazgos: engrosamiento tendinoso, erosión ósea, entesofito, bursitis y señal Power Doppler (PD). Se calculó el score US para entesitis GUESS. El desempeño del score para discriminar entre APs y Ps se evaluó utilizando curvas ROC.
\end{abstract}

Resultados: Se incluyeron 51 sujetos: $A P s=16, P s=15$ y $C S=20$. La edad media fue de $42 \pm 13$ años y el $39 \%$ eran mujeres. La mitad de los pacientes con APs presentaron entesopatía clínica en comparación con ninguno de los otros grupos. Se evaluaron un total de 510 sitios de entesis ( $A P s=160, P S=150, H C=200$ ). Los pacientes con Ps mostraron significativamente una mayor frecuencia de entesofitos, erosiones y PD que los CS. La puntuación media del score GUESS fue diferente entre los grupos (media $\pm D E$ ): $A P s=13 \pm 4, P s=8 \pm 4, C S=3 \pm 2(p<0,01)$. El área bajo la curva $(A B C)$ para el diagnóstico de APs fue de 0,79 (IC95\%=0,63 a 0,95).

Conclusiones: Los pacientes con Ps mostraron una alta frecuencia de entesopatía subclínica en la evaluación US. El score GUESS mostró una alta sensibilidad y moderada especificidad para discriminar entre los pacientes con APs y Ps.

Palabras clave: artritis psoriásica, ultrasonografía.

\section{Abstract}

The aim of our study was to evaluate the prevalence of subclinical enthesopathy in patients with psoriasis (Ps) using ultrasound (US) as a diagnostic tool in daily practice and to evaluate the discriminant capacity of the score to differentiate Ps from Psoriatic Arthritis patients (PsA).

Material and methods: We included healthy controls $(\mathrm{HC})$ patients with Ps and psoriatic arthritis (PsA). Ten enthesis sites were evaluated: insertion of the quadriceps tendon, proximal and distal insertions of the patellar tendon, Achilles tendon and plantar fascia. The US identified the following findings: tendon thickening, bone erosion, enthesophyte, bursitis and PD signal. The GUESS US score was calculated. The score performance to discriminate between APs and Ps was evaluated using ROC curves.

Results: We included 51 subjects: $P s A=16, P s=15$ and $C S=20$. The mean age was $42 \pm 13$ years and $39 \%$ were women. A total of 510 enthesis ( $P s A=160, P s=150, H C=200$ ) were evaluated. Ps patients showed a significantly higher frequency of enthesophytes, erosions and PD than HC. The mean GUESS score was different between groups (mean $\pm S D): P s A=13 \pm 4 \quad P s=8 \pm 4 \quad H C=3 \pm 2(p<0.01)$. The area under the curve (AUC) for the diagnosis of PsA was 0.79 (95\% $\mathrm{Cl}=0.63$ to 0.95 ).

Conclusions: Patients with Ps showed high frequency of subclinical enthesopathy in the US assessment. The GUESS score showed high sensitivity and moderate specificity to discriminate between patients with PsA and Ps.

Key words: psoriatic arthritis, ultrasonography. 


\section{Introducción}

La Psoriasis (Ps) es una enfermedad inflamatoria de la piel que puede afectar hasta al $8 \%$ de la población ${ }^{1}$. La prevalencia de la Artritis Psoriásica (APs) en pacientes con Ps varía de 7,6 a 36\% según las diferentes poblaciones estudiadas ${ }^{2-4}$. Dentro de las características clínicas del compromiso musculoesquelético de la APs, la entesopatía se ubica en un rol central. Propuesta como lesión fundamental en la fisiopatogenia, la lesión inflamatoria de las entesis podría representar el evento inicial en el desarrollo de la enfermedad ${ }^{5-7}$. Teniendo en cuenta la alta frecuencia de síntomas musculoesqueléticos en la población con Ps, las entesopatías, contempladas en los criterios CASPAR para APs, deben ser objetivadas por el reumatólogo en su práctica diaria ${ }^{8}$. Sin embargo, su evaluación clínica es dificultosa y presenta niveles de acuerdo variables entre distintos examinadores ${ }^{9}$. Algunos autores han propuesto el empleo de estudios de imagen de las entesis en pacientes con Ps con el objetivo de detectar con mayor precisión la presencia de $\mathrm{APs}^{10-12}$. Actualmente, la ultrasonografía (US) es utilizada ampliamente por los reumatólogos, y ha demostrado validez y sensibilidad en la evaluación del compromiso entésico ${ }^{13}$. En este estudio nos propusimos utilizar la US para evaluar las entesis de miembros inferiores de pacientes con Ps sin síntomas musculoesqueléticos, comparándolos con pacientes con APs y sujetos sanos, con el objetivo de detectar lesiones subclínicas y evaluar la capacidad discriminativa de la US para diferenciar pacientes con Ps y APs.

\section{Material y métodos}

Se diseñó un estudio de corte transversal que incluyó pacientes consecutivos provenientes de centros de atención ambulatoria de Dermatología (Psoriahué) y Reumatología (Instituto de Rehabilitación Psicofísica). Se les solicitó a los dermatólogos derivar pacientes con diagnóstico confirmado de Ps y que no tuvieran presencia ni antecedentes de síntomas musculoesqueléticos. Adicionalmente, se incluyeron pacientes con APs que debían cumplir criterios CASPAR y el grupo de controles sanos no debían presentar síntomas musculoesqueléticos ni antecedentes de enfermedades reumáticas o Ps. Todos los sujetos incluidos fueron evaluados clínicamente por un reumatólogo experimentado, con el fin de detectar dolor o tumefacción en los sitios de entesis. Se recabaron datos socio-demográficos, clínicos y de las características de enfermedad.

\section{Evaluación US}

El examen US estuvo a cargo de dos reumatólogos ciegos a la exploración clínica y con amplia experiencia en US.
Se evaluaron diez sitios de entesis (de manera bilateral): inserción del tendón del cuádriceps, tendón proximal y distal del ligamento patelar, tendón de Aquiles y fascia plantar. En cada sitio se consignó la presencia de las siguientes lesiones: engrosamiento tendinoso, erosión ósea, entesofito y bursitis, de acuerdo a las definiciones y recomendaciones actuales ${ }^{14-16}$. La entesopatía se definió como la presencia de al menos una lesión US. Luego se calculó el score US para entesitis GUESS (Glasgow Ultrasound Enthesitis Scoring System), que considera un punto ante la presencia de cada lesión en los diez sitios entésicos mencionados, y cuenta con un rango entre $0 \mathrm{y}$ 36 , siendo 36 el puntaje más alto posible ${ }^{14}$. Se evaluó un modelo alternativo al incorporar al GUESS la presencia de señal Power Doppler (PD) en los sitios de entesis, sumando un punto ante la presencia de señal PD en cada entesis, obteniéndose un rango entre 0 y 46.

\section{Análisis estadístico}

Las diferencias entre los grupos se compararon mediante la prueba de chi-cuadrado, y prueba de Kruskal Wallis, según correspondiera. El análisis post hoc se ajustó por el método de Bonferroni. Con el fin de evaluar la capacidad del score de GUESS para discriminar pacientes con Psoriasis cutánea aislada versus Psoriasis cutánea con compromiso articular/entésico (APs), se utilizaron curvas COR (Característica Operativa del Receptor; mejor conocidas por su acrónimo en inglés, curvas ROC, receiver operating characteristic curve), determinando el mejor punto de corte para discriminar estos dos grupos. Debido a que la finalidad de este estudio es evaluar la posible aplicación del score de GUESS para diferenciar pacientes con Psoriasis cutánea de aquellos con APs, no se evaluó la capacidad del score para discriminar pacientes con psoriasis cutánea de la población sana, diferenciación que habitualmente es realizada por el examen clínico del dermatólogo. Un valor de p menor de 0,05 fue considerado estadísticamente significativo.

\section{Resultados}

En total fueron incluidos 51 sujetos: 16 con diagnóstico de APs, 15 con Ps cutánea y 20 sujetos sanos de control. Los datos socio-demográficos y de la enfermedad se muestran en la Tabla 1.

\section{Evaluación entésica}

Se evaluaron clínicamente y por US un total de 510 sitios de entesis (APs=160, Ps=150, controles=200). En el examen clínico, la mitad de los pacientes con APs presentaban dolor y/o tumefacción en sitios de entesis y ninguno en los grupos Ps y de control. Con respecto a la prevalencia total 


\begin{tabular}{|c|c|c|c|c|}
\hline Variable & $\begin{array}{c}\text { Artritis } \\
\text { Psoriásica } \\
(n=16)\end{array}$ & $\begin{array}{c}\text { Psoriasis } \\
(n=15)\end{array}$ & CS $(n=20)$ & $\mathbf{P}^{*}$ \\
\hline Edad, media $\pm D E$ (años) & $50 \pm 14$ & $39 \pm 14$ & $37 \pm 7$ & $<0,01$ \\
\hline Hombres, $\mathrm{n}(\%)$ & & $7(47)$ & $14(70)$ & $>0,20$ \\
\hline $\begin{array}{l}\text { Duración de la psoriasis, } \\
\text { media } \pm D E \text { (años) }\end{array}$ & $16 \pm 10$ & $17 \pm 15$ & $\ldots$ & $>0,20$ \\
\hline $\begin{array}{l}\text { Duración de la artritis } \\
\text { psoriásica, media } \pm D E \text { (años) }\end{array}$ & $5 \pm 7$ & $\ldots$ & ------ & ----- \\
\hline Compromiso axial, n (\%) & $10(63)$ & ------ & ------ & ------ \\
\hline Compromiso periférico, n (\%) & $15(94)$ & ------ & ------ & ------ \\
\hline $\begin{array}{l}\text { Articulaciones dolorosas, } \\
\text { (media } \pm \mathrm{DE})\end{array}$ & $2,3 \pm 2,5$ & ----- & ------ & ------ \\
\hline $\begin{array}{l}\text { Articulaciones inflamadas, } \\
\text { (media } \pm \text { DE) }\end{array}$ & $1,8 \pm 2,3$ & $0 \pm 0$ & $0 \pm 0$ & $<0,01$ \\
\hline Entesopatía clínica, n (\%) & $8(50)$ & $0(0)$ & $0(0)$ & $<0,01$ \\
\hline $\begin{array}{l}\text { Número de sitios afectados } \\
\text { con entesopatía clínica, } \\
\text { (media } \pm D E \text { ) }\end{array}$ & $0,8 \pm 1,1$ & $0 \pm 0$ & $0 \pm 0$ & $<0,01$ \\
\hline $\begin{array}{l}\text { Número de sitios afectados } \\
\text { con entesopatía } \\
\text { ultrasonográfica }{ }^{\star},(\text { media } \pm D E)\end{array}$ & $8,2 \pm 1,5$ & $6,5 \pm 2,4$ & $3,1 \pm 1,7$ & $<0,01$ \\
\hline
\end{tabular}

*Se considera entesopatia ultrasonográfica a la presencia de al menos una lesión entésica.

Tabla 1. Comparación de características clínicas y demográficas entre los grupos.

de entesopatía, la US demostró compromiso entésico en 291 sitios (57\%) mientras que solo en 13 sitios (3\%) fueron detectados por el examen clínico.

\section{Frecuencia de lesiones US en los grupos}

Al considerar el número de sitios con al menos una lesión US (media $\pm \mathrm{DE}$ ), el grupo más afectado fue el APs $(8,2 \pm 1,5)$ seguido por el grupo Ps $(6,5 \pm 2)$ y el grupo control $(3,1 \pm 1,7)(\mathrm{p}<0,01)$. Con respecto a la prevalencia de las diferentes lesiones US en los grupos, las entesis de los pacientes con APs mostraron mayor frecuencia de engrosamiento tendinoso y entesofitos que los pacientes con Ps, mientras que estos presentaron mayor frecuencia de entesofitos, erosiones y PD en comparación con los controles (ver Tabla 2).

\section{Desempeño del Score GUESS}

La media del score GUESS fue significativamente mayor en los pacientes con Ps comparado con los controles: $8 \pm 4$ vs. $3 \pm 2(\mathrm{p}<0,01)$. A su vez, la media del score GUESS fue mayor en el grupo con APs $(13 \pm 4)$ comparado con los otros dos grupos $(\mathrm{p}<0,01)$.

En el análisis por curvas ROC, el área bajo la curva (ABC) para discriminar pacientes con Ps de aquellos con APs fue de 0,79 (IC95\%=0,63 a 0,95) (ver Gráfico 1). Al evaluar distintos puntos de corte del score GUESS para el diagnóstico de APs se observó que un valor $\geq 8$ mostró una sensibilidad de $94 \%$ y una especificidad de $60 \%$, con un Likelihood Ratio positivo de 2,34 (ver Tabla 3). $\mathrm{La}$ adición de PD no tuvo un impacto significativo en la capacidad discriminante del score GUESS (ABC 0,78; IC $95 \%=0,62-0,95)$.

\begin{tabular}{|c|c|c|c|}
\hline $\begin{array}{c}\text { Punto de Corte- } \\
\text { Score GUESS }\end{array}$ & Sensibilidad & Especificidad & $\begin{array}{c}\text { Likelihood } \\
\text { Ratio + }\end{array}$ \\
\hline$\geq 7$ & $100 \%$ & $40 \%$ & 1,67 \\
\hline$\geq 8$ & $94 \%$ & $60 \%$ & 2,34 \\
\hline$\geq 10$ & $81 \%$ & $60 \%$ & 2,03 \\
\hline$\geq 12$ & $63 \%$ & $73 \%$ & 2,34 \\
\hline$\geq 13$ & $50 \%$ & $73 \%$ & 1,88 \\
\hline$\geq 14$ & $44 \%$ & $87 \%$ & 3,28 \\
\hline$\geq 15$ & $38 \%$ & $87 \%$ & 2,81 \\
\hline$\geq 16$ & $38 \%$ & $93 \%$ & 5,63 \\
\hline$\geq 17$ & $31 \%$ & $93 \%$ & 4,69 \\
\hline$\geq 20$ & $6 \%$ & $100 \%$ & 5,31 \\
\hline
\end{tabular}

Tabla 3. Sensibilidad y Especificidad según diferentes puntos de corte del Score GUESS para el diagnóstico de artritis psoriásica.

\section{Discusión}

Uno de los principales hallazgos de este estudio consiste en la demostración de una alta prevalencia de alteraciones entésicas detectadas por US en pacientes con Ps cutánea sin manifestaciones clínicas de APs. Estos pacientes presentaron una media de más de 6 sitios entésicos comprometidos en la evaluación por US y ninguno de estos reveló síntomas en el examen clínico. Adicionalmente, los pacientes con Ps presentaron un número más alto de lesiones y de puntajes GUESS al compararlos con los controles sanos. Hallazgos similares han sido reportados

\begin{tabular}{|l|c|c|c|}
\hline Lesión US & $\begin{array}{c}\text { Artritis Psoriásica } \\
\text { (n de entesis=160) }\end{array}$ & $\begin{array}{c}\text { Psoriasis } \\
\text { (n de entesis=150) }\end{array}$ & $\begin{array}{c}\text { Control } \\
\text { (n de entesis=200) }\end{array}$ \\
\hline $\begin{array}{l}\text { Engrosamiento } \\
\text { tendinoso, } \mathrm{n}(\%)\end{array}$ & $77(48)$ & $38(25)$ & $38(19)$ \\
\hline Entesofito, $\mathrm{n}(\%)$ & $88(55)$ & $65(43)$ & $20(10)$ \\
\hline Bursitis, $\mathrm{n}(\%)$ & $18(11)$ & $8(5)$ & $2(3)$ \\
\hline Erosión, $\mathrm{n}(\%)$ & $24(15)$ & $14(9)$ & $<0,01^{\mathrm{a}}$ \\
\hline Power Doppler, $\mathrm{n}(\%)$ & $21(13)$ & $11(7)$ & $<0,05^{\mathrm{b}}$ \\
\hline
\end{tabular}

a Diferencia significativa entre APs vs. Ps y controles.

${ }^{b}$ Diferencia significativa entre los tres grupos.

c Diferencia significativa entre APs vs. controles.

${ }^{\mathrm{d}}$ Diferencia significativa entre APs y Ps vs. controles.

${ }^{\mathrm{e}}$ Diferencia significativa entre APs y Ps vs. controles.

Tabla 2. Descripción de los hallazgos ultrasonográficos en 510 sitios de entesis. 


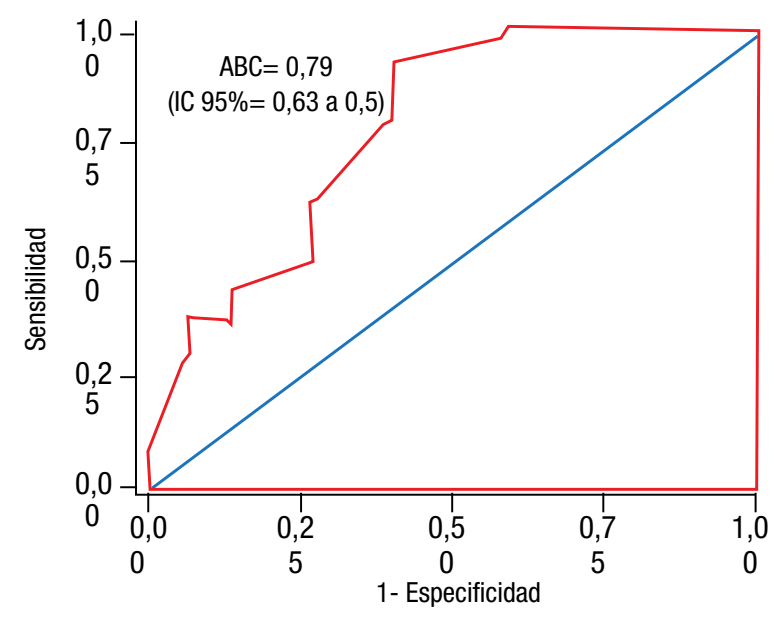

\section{Gráfico 1. Área bajo la curva del GUESS para el diagnóstico de artritis psoriásica en pacientes con psoriasis.}

por otros autores ${ }^{10,17,18}$. Gisondi y cols., en un estudio de US similar al nuestro, estudió comparativamente las entesis de pacientes con Ps y de sujetos sanos, demostrando una alta prevalencia de entesopatía subclínica y mayores puntuaciones del score GUESS en el grupo con $\mathrm{Ps}^{17}$. La evidencia proporcionada por estudios de imagen en sujetos con Ps sin artritis establecida apoya la hipótesis de una mayor susceptibilidad al stress biomecánico en las entesis de estos pacientes. Según lo propuesto por Mc Gonagle y cols., las entesis, estructuras habitualmente expuestas a un proceso fisiológico de micro daño y reparación, tendrían una respuesta anormal en los pacientes con Ps, favoreciendo la aparición de mecanismos inflamatorios que no logran autolimitarse y que además podrían explicar la fisiopatogenia de la $\mathrm{APs}^{7}$.

Nuestros resultados demuestran la utilidad de la valoración US del daño entésico y su capacidad para identificar pacientes con APs dentro de la población con Ps, y coinciden con lo publicado en estudios previos ${ }^{17,19}$. Estos reportes confirman la capacidad de la US para la evaluación de las entesis en pacientes con Ps, y plantean a su vez la posibilidad de utilizar estos datos con fines pronósticos, sobre todo considerando que la US es una técnica simple, accesible y capaz de implementarse como herramienta de screening. Sin embargo, el valor predictivo de estos hallazgos US para el desarrollo de APs deberían ser confirmados en estudios prospectivos. En esta línea de investigación, el único estudio con seguimiento longitudinal de pacientes con Ps publicado al presente reporta que los valores basales altos del score GUESS y la presencia de engrosamiento tendinoso actuaron como predictores de desarrollo de $\mathrm{APs}^{20}$.

Cabe aclarar que nuestro estudio cuenta con algunas limitaciones, siendo la principal el bajo número de pacientes incluidos. Además, no se incluyó el análisis de la correlación intra o interobservador, aunque debe aclararse que los dos ecografistas a cargo de las evaluaciones cuentan con amplia experiencia en la evaluación ecográfica de las entesis. Otra limitación que debe mencionarse es que no se han considerado algunos factores capaces de influir en las entesopatías, como el índice de masa corporal, la actividad física y enfermedades endócrino-metabólicas ${ }^{21,22}$.

\section{Conclusiones}

La evaluación por US de las entesis de los pacientes con Ps reveló una alta frecuencia de entesopatía subclínica y el empleo sistemático del score de GUESS podría ser de utilidad para la identificación precoz de enteropatía en este grupo de pacientes. A su vez, este score podría emplearse como prueba diagnóstica complementaria para el diagnóstico precoz de APs en población con Ps cutánea, sin embargo esto deberá ser confirmado por estudios de seguimiento longitudinal.

\section{Bibliografía}

1. Parisi R, Symmons DP, Griffiths CE, Ashcroft DM. Identification and Management of Psoriasis and Associated ComorbidiTy (IMPACT) project team. Global epidemiology of psoriasis: a systematic review of incidence and prevalence. J Invest Dermatol. 2013; 133: 377-85.

2. Shbeeb M, Uramoto KM, Gibson LE, O'Fallon WM, Gabriel SE. The epidemiology of psoriatic arthritis in Olmsted County, Minnesota, USA, 1982-1991. J Rheumatol. 2000; 27:1247-50.

3. Barisic-Drusko V, Dobric I, Pasic A, Paljan D, Jukic Z, Basta- Juzbasic A, et al. Frequency of psoriatic arthritis in general population and among the psoriatics in department of dermatology. Acta Derm Venereol. 1994; 186:107-8.

4. Gisondi P, Girolomoni G, Sampogna F, Tabolli $S$, Abeni D. Prevalence of psoriatic arthritis and joint complaints in a large population of Italian patients hospitalised for psoriasis. Eur J Dermatol. 2005;15:279-83.

5. McGonagle D, Gibbon W, Emery P. Classification of inflammatory arthritis by enthesitis. Lancet. 1998;352:1137-40.

6. McGonagle D, Gibbon W, O'Connor P, Green M, Pease C, Emery P. Characteristic magnetic resonance imaging entheseal changes of knee synovitis in spondylarthropathy. Arthritis Rheum. 1998; 41: 694700.

7. McGonagle D, Tan AL, Benjamin M. The 
biomechanical link between skin and joint disease in psoriasis and psoriatic arthritis: what every dermatologist needs to know. Ann Rheum Dis. 2008; 67: 1-4.

8. Taylor W, Gladman D, Helliwell P, Marchesoni A, Mease P, Mielants H; CASPAR Study Group. Classification criteria for psoriatic arthritis: development of new criteria from a large international study. Arthritis Rheum. 2006; 54: 2665-73.

9. Gladman DD, Inman RD, Cook RJ, et al. International spondyloarthritis interobserver reliability exercisethe INSPIRE study: II. Assessment of peripheral joints, enthesitis, and dactylitis. J Rheumatol 2007;34:1740-5.

10. Naredo E, Möller I, de Miguel E, Batlle-Gualda E, Acebes C, Brito E, et al. High prevalence of ultrasonographic synovitis and enthesopathy in patients with psoriasis without psoriatic arthritis: a prospective case-control study. Rheumatology (Oxford). 2011; 50: 1838-48.

11. Simon D, Faustini F, Kleyer A, Haschka J, Englbrecht $\mathrm{M}$, Kraus S, et al. Analysis of periarticular bone changes in patients with cutaneous psoriasis without associated psoriatic arthritis. Ann Rheum Dis. 2016; 75: 660-6.

12. Faustini F, Simon D, Oliveira I, Kleyer A, Haschka J, Englbrecht M, et al. Subclinical joint inflammation in patients with psoriasis without concomitant psoriatic arthritis: a cross-sectional and longitudinal analysis. Ann Rheum Dis. 2016 Feb 25.

13. Mata Arnaiz MC, de Miguel Mendieta E. Usefulness of ultrasonography in the assessment of peripheral enthesis in spondyloarthritis. Reumatol Clin. 2014; 10: 113-9.

14. Balint PV, Kane D, Wilson H, McInnes IB, Sturrock $\mathrm{RD}$. Ultrasonography of entheseal insertions in the lower limb in spondyloarthropathy. Ann Rheum Dis. 2002; 61: 905-10.

15. Wakefield RJ, Balint PV, Szkudlarek M, Filippucci E, Backhaus M, D'Agostino MA, et al. Musculoskeletal ultrasound including definitions for ultrasonographic pathology. J Rheumatol. 2005; 32: 2485-7.

16. De Miguel E, Cobo T, Muñoz-Fernández S, Naredo E, Usón J, Acebes JC, et al. Validity of enthesis ultrasound assessment in spondyloarthropathy. Ann Rheum Dis. 2009; 68:169-74.

17. Gisondi P, Tinazzi I, El-Dalati G, Gallo M, Biasi D, Barbara LM, et al. Lower limb enthesopathy in patients with psoriasis without clinical signs of arthropathy: a hospital-based case-control study. Ann Rheum Dis. 2008; 67: 26-30.

18. Gutierrez M, Filippucci E, De Angelis R, Salaffi F, Filosa G, Ruta S, et al. Subclinical entheseal involvement in patients with psoriasis: an ultrasound study. Semin Arthritis Rheum. 2011; 40: 407-12.
19. Eder L, Jayakar J, Thavaneswaran A, Haddad A, Chandran V, Salonen D, et al. Is the Madrid Sonographic Enthesitis Index useful for differentiating psoriatic arthritis from psoriasis alone and healthy controls? J Rheumatol. 2014; 41: 466-72.

20. Tinazzi I, McGonagle D, Biasi D, Confente S, Caimmi C, Girolomoni G, et al. Preliminary evidence that subclinical enthesopathy may predict psoriatic arthritis in patients with psoriasis. J Rheumatol. 2011; 38: 2691-2.

21. Schweitzer ME, Karasick D. MR imaging of disorders of the Achilles tendon. AJR Am J Roentgenol. 2000; 175: 613-25.

22. Civeira F, Castillo JJ, Calvo C, Ferrando J, de Pedro C, Martínez-Rodés P, et al. Achilles tendon size by high resolution sonography in healthy population. Relationship with lipid levels. Med Clin (Barc). 1998; 111:41-4. 